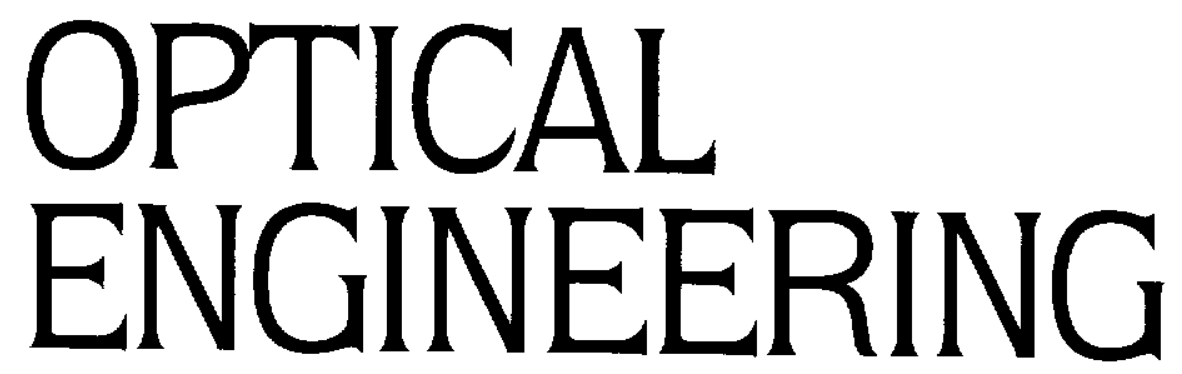

October 2000

Volume 39 Number 10

ISSN 0091-3286

SPIE-

The International

Society for

Optical Engineering

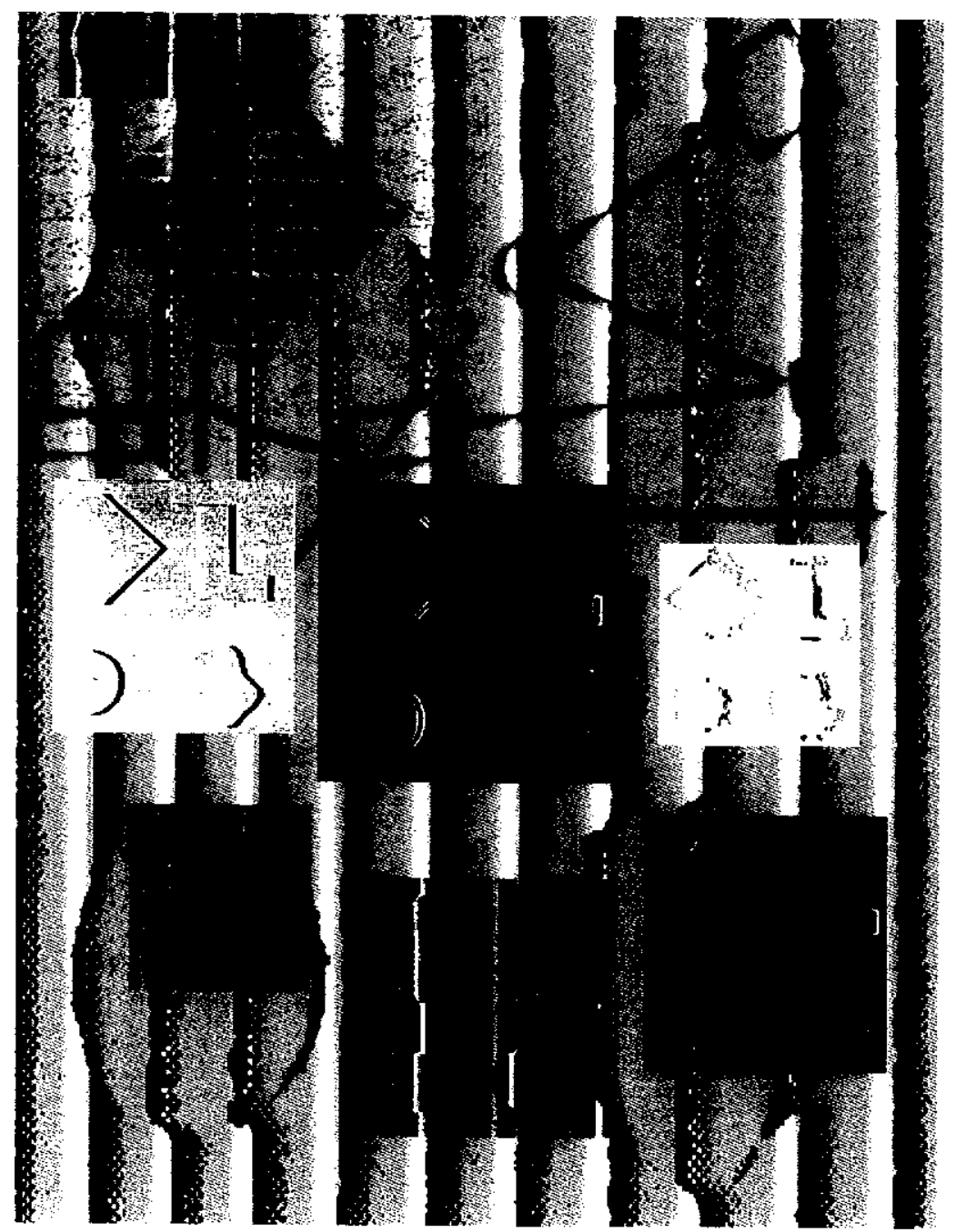

I Featuring Papers on

Correlators

Detection

Detectors

Discrete Transforms

Echo Rating

Imaging

Interferometry

Lasers

Lenses

Measurement

Moiré

Neural Networks

Non-imaging Optics

Optical Components

Ray Tracing

Sensors

Signal Processing

Single Crystal Fibers

Wavelets

I Communications

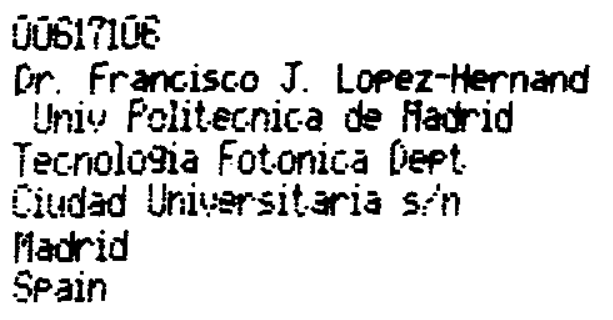

E-28ưtu 
2763 Adaptive optics systems implemented using two-dimensional phase retrieval and a microelectromechanical

deformable mirror

Stanley Rogers, Frank Scarpino, Tommy Williamson, Max K. Lee,

Jason Baumbach, William D. Cowan, Donald R. Wiff

\section{RAY TRACING}

2775 Ray-tracing algorithms for fast calculation of the channel impulse response on diffuse IR wireless indoor channels Francisco J. López-Hernández, Rafael Pérez-Jiménez, Asunción Santamaría

\section{SENSORS}

2781 Six band multispectral sensor using off-axis three-mirror reflective optics

Kunihiro Tanikawa, Toshihiro Okamura, Jun-ichi Kudo, Hideo Wada, Hiromichi Shirahata

SIGNAL PROCESSING

2789 Significance of the recovery filter in deconvolution from wavefront sensing

Justo Arines, Salvador Bará

2797 Novel adaptive digital signal processing algorithm for a stationary reticle seeker

Jeong-Su Oh, Kyung-Soo Doo, Surng-Gabb Jahng, Dong-Sun Seo, Jong-Soo Choi

\section{SINGLE CRYSTAL FIBERS}

2804 Second-harmonic generation using an $a$ axis $\mathrm{Nd:MgO}: \mathrm{LiNbO}_{3}$ single crystal fiber with $\mathrm{Mg}$-ion indiffused cladding

Wenxiu Que, Y. Zhou, Y. L. Lam, Y. C. Chan, C. H. Kam, Yujing Huo, Xi Yao

WAVELETS

2810 New method for designing two-channel causal stable IIR perfect reconstruction filter banks and wavelet bases

J. S. Mao, S. C. Chan, K. L. Ho

2821 Wavelet transform for detection of partial fringe patterns induced by defects in nondestructive testing of holographic interferometry and electronic speckle pattern interferometry

Xide Li

\begin{tabular}{ll}
\hline COMMUNICATIONS \\
\hline $2828 \quad \begin{array}{l}\text { Object recognition using three-dimensional correlation of } \\
\text { range images } \\
\text { Jáder Guerrero-Bermúdez, Jaime Meneses, Oscar Gualdrón }\end{array}$
\end{tabular}

2831 Experimental realization of sculptured-thin-film polarizationdiscriminatory light-handedness inverters Ian J. Hodgkinson, Akhlesh Lakhtakia, Qi hong Wu 


\section{Ray-tracing algorithms for fast calcultion of the channel impulse response on diffuse IR
wireless indoor channels}

Francisco J. López-Hernández, MEMBEA SPIE

Universidad Politécnica de Madrid

E.T.S.I.T. Dpto. T. Fotónica

Ciudad Universitaria $\mathbf{s} / \mathbf{n}$

28040 Madrid, Spain

E-mail: dxtn@tfo.upm.es

\author{
Rafael Pérez-Jiménez \\ E.U.I.T.T. Grupo de Tecnología Fotónica y
Comunicaciones \\ D. SS.CC., ULPGC \\ Campus de Tafira \\ 35017, Las Palmas de Gran Canaria, Spain
}

\author{
Asunción Santamaría \\ Universidad Politécnica de Madrid \\ E.T.S.I.T. Dpto. Electrm. y T. Circuitos \\ Ciudad Universitaria $s / n$ \\ 28040 Madrid, Spain
}

\begin{abstract}
A modified Monte Carlo algorithm for the calculetion of the impulse response on infrared wireless indoor for the calculation of the is well known, the characteristics of the room channels is presented. As nel is implemented can lead to problems in commune IP diffuse chanmultipath penalty on the maximum baud rate communication, such as a Classical algorithms require large computation hidden-station situations. impulse response in an ordinary-size room. sibility of validating the assumptions made for the Carlo offers the pos(basically, the Lambertian nature of all reflections) with assic algorithms complexity that is determined by the reflections) with a computational have developed a mixed ray-tracing-deterministic alged by the user. We that each ray contributes to the fina-deterministic algorithm that assures times as it rebounds with an obstacle. It increases fre function as many ber of contributions and reduces, to the increases dramatically the numfor an accurate simulation. Extensive simulation extent, the time required and are compared with those of strate that the method presented here is much fathods. We demonclassical simulation schemes. It here is much faster than Monte Carlo in itself or as a validation algorithm for used as a method of simulation broadening. (c) 2000 society of Photo-Optical intrumparative studies of pulse [50091-3286(00)00909-0]
\end{abstract}

Subject terms: simulation; Monte Carlo; wireless optical transmission; ray tracing. Paper 990431 received Nov. 5, 1999; revised manuscript received Feb. 25, 2000;
accepted for publication Mar. 8,2000 . 
López-Hernández, Pérez-Jīménez, and Santamaria: Ray-tracing algorithms . . .

Table 1 Basic algorithm.

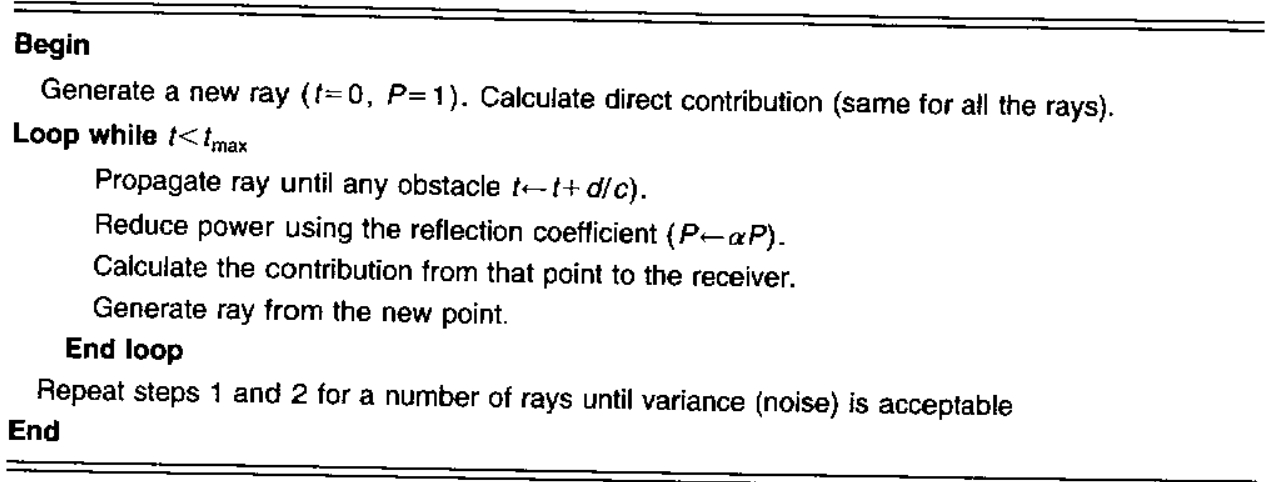

such as windows or mirrors, the reflection is dominant over scattering at any angle. Our model implements both diffuse (Lambert) and mirror reflections, so it can simulate real rooms more closely than the deterministic ones can.

\section{Description}

This is a ray-tracing method. ${ }^{5}$ Many rays are generated at the emitter position, having a distribution probability equal to the emission profile, or angular optical intensity function. When a ray impinges on an obstacle (wall, ceiling, etc.), the point where it reaches the obstacle is converted in a new optical source; thus a new ray is generated, and the process continues until the time of flight (counted from the generation on the emitter) reaches the maximum time to simulate $\left(t_{\max }\right)$.

After every reflection the power of the ray is reduced by the reflection coefficient of the obstacle, so two variables are associated with every ray: the time from its generation, and the power it carries.

In a Monte Carlo simulation, there is a tiny probability for the ray reaching the receiver before the maximum simulation time. ${ }^{6}$ When this happens, the power carried by the ray is detected with the delay of the trip. But the probability of this is very low, about $10^{-7}$, so many millions of rays have to be generated to get a reliable result. The chances are very different if we use the fact that we know the contribution of a scatterer surface to the receiver. In this way, when the ray impinges on an obstacle, not only is a new ray generated, but the reflected power contribution to the receiver is calculated. Thus, we have changed from using only one of many of million of rays, ${ }^{7}$ to using every ray generated several times (as many as there are reflections). Of course, before generating a new ray, the direct emitterto-receiver contribution is added up.

The algorithm is presented in Table 1. Next we will describe the equations used at every step.

\subsection{Emitter}

The emitter is defined by its position and orientation $\left(\mathbf{r}_{e}, \hat{\mathbf{n}}_{e}\right)$ and its intensity profile $I(\theta)$, where cylindrical symmetry around the normal to the emitting surface has been assumed. Every launched ray will have a normalized unit power. After the simulation, this allows the renormalization of the power emitted by all of the rays. As the system is linear and time-invariant, there is no problem in doing so. If several emitters with different powers are to be simulated, superposition can be applied.

The main problem with the emitter is generating rays following a given intensity profile. The most used approximation is the extended Lambertian profile

$I=I_{0} \cos ^{n} \theta$,

where the exponent defines the width of the beam and $I_{0}$ is a normalization constant. For $n=1$, the Lambertian scattering profile is obtained. The most significant characteristic of the extended Lambertian is that the maximum intensity direction is always normal to the emitter surface. Several device cases or arrays do not fulfill this condition, and for them this model is useless. So both cases, extended Lambertian and general, have been studied:

1. Extended Lambertian: This case includes Lambertian scattering $(n=1)$. The axis definition is given in Fig. 1 , where the emitter surface is the $X Y$ plane. As defined in Eq. (1), the main random variable is $\theta$. We use the cumulative probability, i.e., the probability of a ray having a $\theta$ value lower or equal to $\Theta$, given by

$$
P(\theta \leqslant \Theta)=\int_{0}^{2 \pi} \mathrm{d} \varphi \int_{0}^{\Theta} I(\theta) \sin \theta \mathrm{d} \theta=1-\cos ^{n+1}(\Theta) .
$$


We use the components $r$ and $z$, which are

$r=\sin \theta, \quad z=\cos \theta$.

Since $z$ and $\theta$ are decreasing monotonic functions of one another, we have

$$
\begin{aligned}
& P(z \geqslant Z)=P(\theta \leqslant \Theta), \\
& P(z \leqslant Z)=1-P(z \geqslant Z)=Z^{n+1} .
\end{aligned}
$$

To get a random unit vector of which the $z$ component has a cumulative probability given by Eq. (4) two random numbers are generated having a uniform probability in the range $[0,1)$. Let $u$ and $v$ be these numbers; then the sequence of calculations is

$z=\sqrt[n+1]{u}, \quad r=\sqrt{1-z^{2}}$,

$x=r \cos (2 \pi v), \quad y=r \sin (2 \pi v)$,

where $x, y$, and $z$ are the components of a unit vector relative to a coordinate system normal to emitter surface.

2. General profile: Let be $I(\theta)$ a tabulated or analytical function. As before,

$P(z \leqslant Z)=1-2 \pi \int_{1}^{a \cos Z} I(\theta) \sin \theta \mathrm{d} \theta=K \cdot F(Z)$.

Equations (l) and (6) produce a function $F(Z)$, which should be normalized using the constant $K$ to get $F(1)=1$. If the inverse of $F(Z)$ is applied to a random number, generated with uniform probability density in $[0,1)$, the required $z$ component is achieved. The other components, $x$ and $y$, are generated from $z$ using the last two equations in (5).

In both cases the unit vector is based on a coordinate systern normal to the emitter surface. It needs to be transformed into room coordinates. This is achieved by using the matrix $\boldsymbol{M}_{e}$ given in

$$
\begin{aligned}
\hat{\mathbf{n}}_{e}=\left[\begin{array}{l}
n_{e x} \\
n_{e y} \\
n_{e z}
\end{array}\right]_{\text {room }} & {\left[\begin{array}{ccc}
-\sin \phi & \cos \phi & 0 \\
-\cos \phi \cos \theta & -\sin \phi \cos \theta & \sin \theta \\
\cos \phi \sin \theta & \sin \phi \sin \theta & \cos \theta
\end{array}\right] } \\
& \times\left[\begin{array}{l}
x \\
y \\
z
\end{array}\right]_{\text {emitter }}=\mathbf{M}_{e}\left[\begin{array}{l}
x \\
y \\
z
\end{array}\right]_{\text {emitter }} .
\end{aligned}
$$

We have arbitrarily chosen the $X$ axis of the emitter to be on the $X Y$ plane of the room.

\subsection{Receiver}

The receiver (photodiode plus receiving optics) is defined by its position and orientation $\left(\mathbf{r}_{r}, \hat{\mathbf{n}}_{r}\right)$, detecting surface $\left(A_{r}\right)$, and FOV, which is the maximum angular deviation from the normal for a ray to be detected.

As stated before, any time a ray impinges on an obstacle, the contribution from that point to the receiver is calculated. If the obstacle is placed at $\mathbf{r}$ and its surface normal is $\hat{\mathbf{n}}$, the contribution is given by (a)

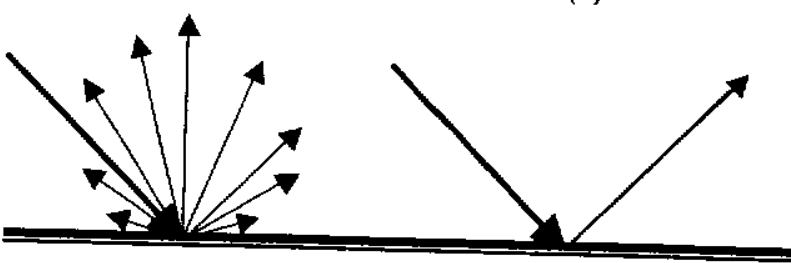

Fig. 2 (a) Scattering and (b) mirror reflection. where

$\mathbf{d}=\mathbf{r}_{r}-\mathbf{r}, \quad d=|\mathbf{d}|$,

$\phi=\operatorname{angle}(\mathbf{n}, \mathbf{d}), \quad \psi=\operatorname{angle}\left(\mathbf{n}_{r}, \mathbf{d}\right)$,

where $d$ is the distance described by the ray. The factor $\cos \phi$ is valid for Lambertian diffuse reflections; for the direct emitter contribution it has to be changed according to the emitter intensity profile.

The power contribution $P_{r}$ is delayed by $d / c$, a quantity to be added to the time accumulated for that ray.

\subsection{Obstacles}

When a ray impinges on an obstacle, two kinds of reflection are possible: mirror (MR) and diffuse (DR). (See Fig. 2.) In a general case, both of them take place simultaneously. In the model, only one new ray is generated, and the choice between MR or DR is based on a probability that depends on the surface characteristics and incidence angle. For example, a glass window will have an MR probability equal to one (diffuse reflection neglected), while a white area on the ceiling will scatter the light except for grazing incidence angles (probability of MR almost 0 for incidence angles lower than $50 \mathrm{deg}$ ).

For every different area of each obstacle surface an MR threshold function is defined. This function depends, in general, on the incidence angle of the ray. When a ray arrives at the obstacle, a random number in the range $[0,1)$ is generated; after comparing this number with the MR threshold, DR or MR is used. The output direction is calculated as follows

- Mirror reflection (MR): The incident and reflected rays are in the same plane and form the same angle with the normal to the surface. If $\hat{\mathbf{n}}_{i}, \hat{\mathbf{n}}_{\mathbf{0}}$, and $\hat{\mathbf{n}}_{s}$ are the unit vectors defining, respectively, the incident, reflected, and surface normal directions, that law can be expressed as

$\hat{\mathbf{n}}_{o}=\hat{\mathbf{n}}_{i}-2\left(\hat{\mathbf{n}}_{i} \cdot \hat{\mathbf{n}}_{s}\right) \hat{\mathbf{n}}_{s}$.

- Diffuse reflection (DR): The output direction is calculated using $\mathrm{Eq}$. (5), with $n=1$. 
López-Hernández, Pérez-Jiménez, and Santamaría: Ray-tracing algorithms . . .

Table 2 Data used in simulations.

\begin{tabular}{|c|c|c|}
\hline \multirow[b]{2}{*}{ Parameter } & \multicolumn{2}{|c|}{ Value } \\
\hline & A & $\mathrm{B}$ \\
\hline \multicolumn{3}{|l|}{ Room: } \\
\hline Length $x(m)$ & 5 & 7.5 \\
\hline Width $y(m)$ & 5 & 5.5 \\
\hline Height $z(\mathrm{~m})$ & 3 & 3.5 \\
\hline \multicolumn{3}{|l|}{ SR coefficients: } \\
\hline$\rho_{1}$ & 0.8 & 0.58 \\
\hline$\rho_{2}$ & 0.8 & 0.56 \\
\hline$\rho_{3}$ & 0.8 & 0.3 \\
\hline$\rho_{4}$ & 0.8 & 0.12 \\
\hline$\rho_{5}$ & 0.8 & 0.69 \\
\hline$\rho_{6}$ & 0.3 & 0.09 \\
\hline \multicolumn{3}{|l|}{ Emitter: } \\
\hline Beam width & 1 & 1 \\
\hline \multicolumn{3}{|l|}{ Position $\mathbf{r}_{e}(\mathrm{~m})$} \\
\hline$x$ & 2.5 & 5.0 \\
\hline$y$ & 2.5 & 1.0 \\
\hline$z$ & 3 & 3.3 \\
\hline \multicolumn{3}{|c|}{ Orientation $\mathbf{n}_{e}$ (deg) } \\
\hline$\varphi$ & 0 & 10 \\
\hline$\theta$ & -90 & -70 \\
\hline \multicolumn{3}{|l|}{ Receiver: } \\
\hline Area $\left(\mathrm{cm}^{2}\right)$ & 1 & 1 \\
\hline \multicolumn{3}{|l|}{ Position $\mathbf{r}_{e}(\mathbf{m})$} \\
\hline$x$ & 0.5 & 2.0 \\
\hline$y$ & 1.0 & 4.0 \\
\hline$z$ & 0 & 0.8 \\
\hline \multicolumn{3}{|l|}{ Orientation $\mathbf{n}_{e}$ (deg) } \\
\hline$\varphi$ & 0 & 0 \\
\hline$\theta$ & 90 & 90 \\
\hline FOV (deg) & 85 & 70 \\
\hline
\end{tabular}

Two reflection coefficients are defined, one for DR and one for MR. The power of the input ray is multiplied by the appropriate coefficient to get the output power. Usually the MR coefficient is much larger than the DR.

\subsection{Propagation Between Reflections}

Let $\mathbf{r}_{0}$ be the starting position of a ray (either the emitter position or where the last collision happened), and $\hat{\mathbf{n}}$ the direction generated by Eq. (5) or (9). The vector equation describing the ray flight is ${ }^{5}$

$\mathbf{r}(\lambda)=\mathbf{r}_{0}+\lambda \hat{\mathbf{n}}$

where $\lambda$ is the distance the ray has traveled. For several obstacles, Eq. (10) needs to be solved together with the equations describing them. In general, a set of $\lambda$ values are obtained, one for every obstacle. It is evident that the small
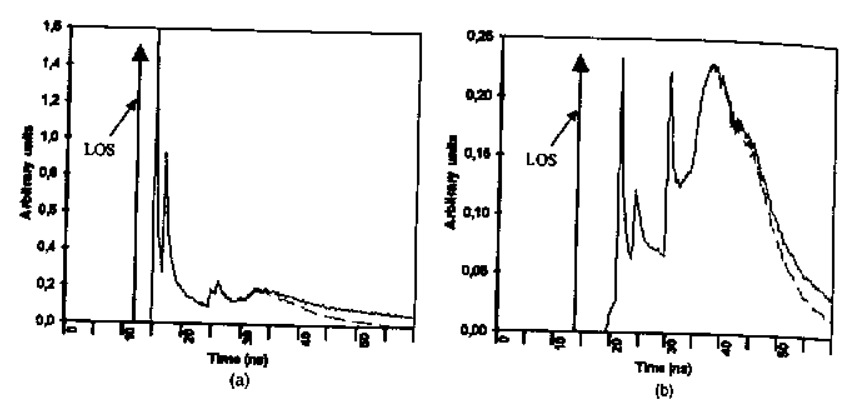

Fig. 3 Simulation results for configurations (a) and (b) (data in Table 2). Dashed curves: deterministic approach; solid curves: the
Monte Carlo simulation.

positive value of $\lambda$ is the first obstacle reached by the ray. The quantity $\lambda / c$ has to be added to the accumulated trave time of the ray.

Using Eq. (10), the new starting point is calculated, and the obstacle characteristics are used to generate, if needed, a new ray.

\section{Results}

The algorithm described in Sec. 2 was implemented in a Pentium II PC, using Microsoft Visual Basic. The choice of an interpreted language, instead of Fortran or C, is due to the ease of changing data or code during the execution of the program. Usually this is at the expense of longer execution times. Nevertheless, the algorithm proposed is fast enough to get one simulation in several minutes, which is twenty times faster than previous methods (implemented in Fortran or C).

Two room configurations are presented as examples: configurations $\mathrm{A}$ and $\mathrm{B}$. The data used in the examples are in Table 2. Basically the scenarios are empty rooms where multiple reflections occur on the walls, ceiling, and floor. Only DR is considered, so as to be able to compare the simulations with previous algorithms unable to simulate MR. These examples are the same as those published using other methods, ${ }^{2,3}$ so they can be compared with them. Figure 3 presents the impulse responses obtained. It is noticeable that the tails of the impulses are larger than that obtained in Ref. 2. This is because in that algorithm the number of reflections was limited to 3 or 4 , while our method is able to calculate as many reflections as needed. In fact, we originally reserved space for 20 reflections, but once a ray reached this limit the program could not cope with it, so to test for this, we keep a record of the contributions not only by the delay, but also by the reflection number.

It is possible to calculate the contribution of each rebound to the total impulse response; the contributions of the first steps are presented in Fig. 4. The vertical units are arbitrary, but the scale is the same for all of them, so their relative importance can be seen from the values in the graphs.

It is also noticeable that for the same number of rays, the noise increases with the number of rebounds. Fortunately, the influence of the later rebounds on the final result is lower because of their low power contribution. Further refinement can be obtained by numerical filtering methods, such as moving averaging. In Fig. 5 is presented the evo- 

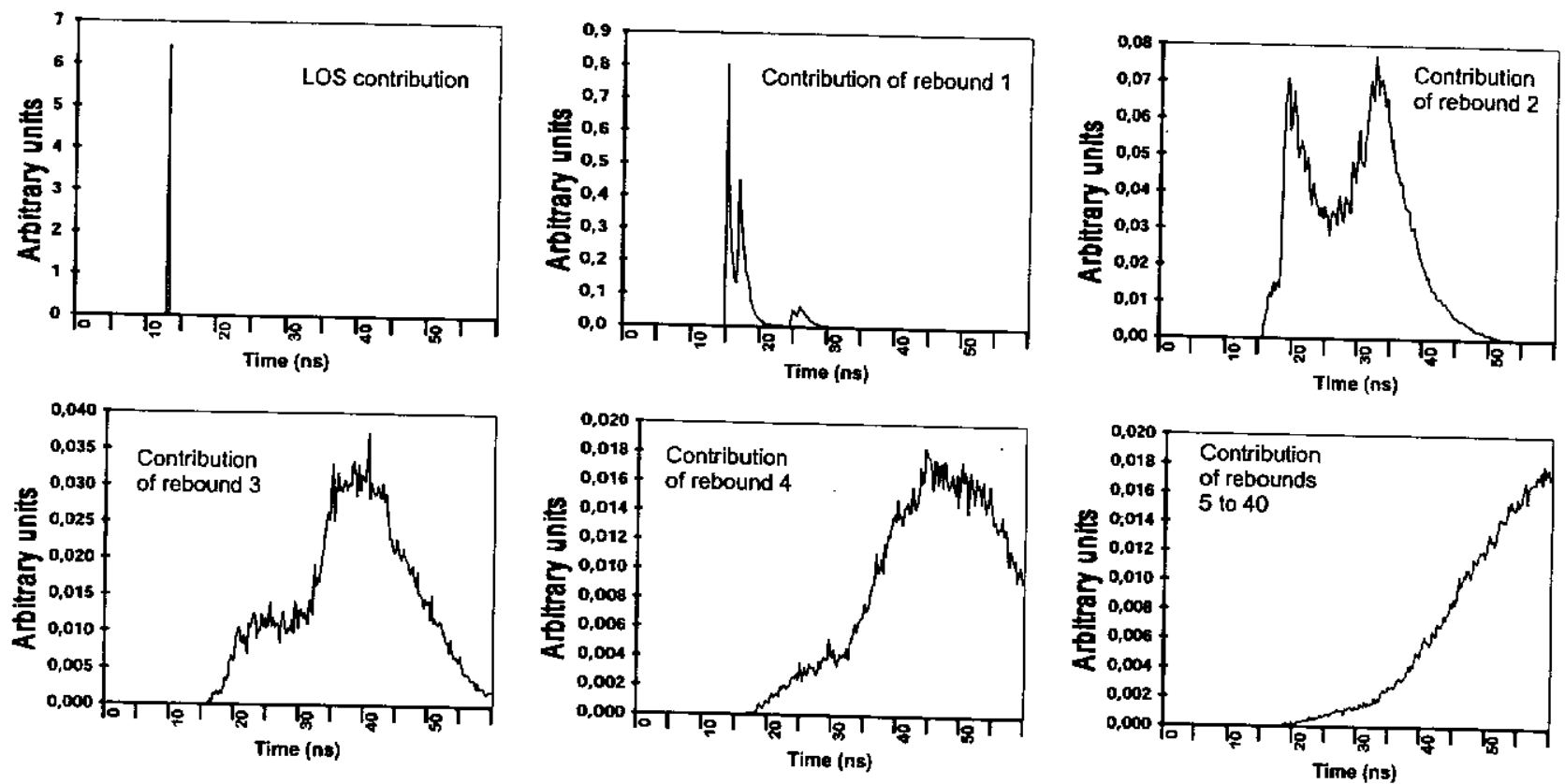

Fig. 4 Relative contribution according to the number of rebounds.

lution of the impulse response for different number of rays. It can be seen the variance reduction as the number of rays increases. The vertical axis units are arbitrary, the $x$ axis is in nanoseconds, and the graph is from configuration $A$ without line-of-sight (LOS) contribution.

Once the impulse response is found, the room (with the emitter and receiver) is completely characterized in terms of bandwidth, and the effect produced by the room on any signal waveform can be obtained, because the room acts as a linear and time-invariant low-pass filter. This assumption can be made because the transmitted baud rate is much faster than the variations in the room configuration.

\section{Conclusions}

A very fast method for simulating multipath response has been presented. Its implementation using compiled languages will improve its performance and allow comparative studies on the effects of surface characteristics, optimi-
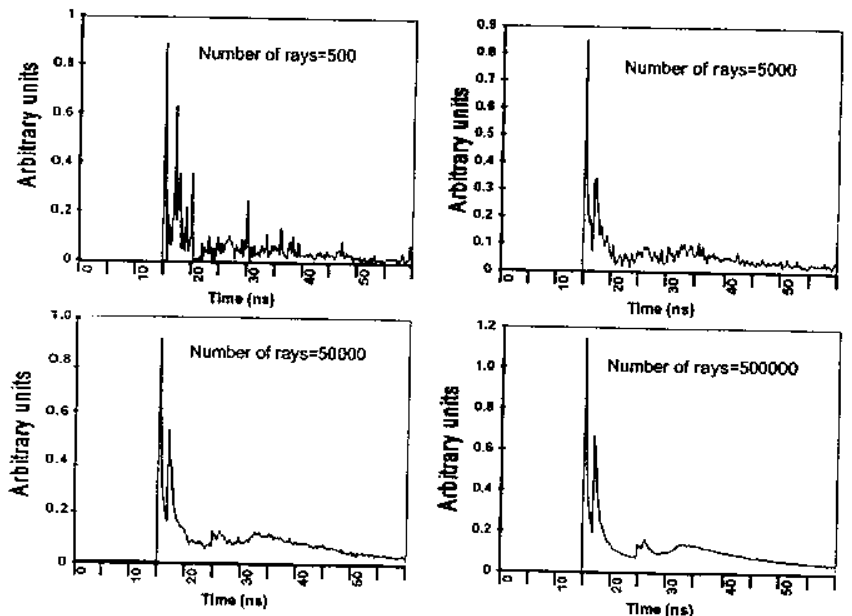

Fig. 5 Variance reduction with increasing number of rays. zation of emitter and receiver orientations, etc. Another important project is the study of the effect of multipath dispersion on intersymbol interference (ISI) and the penalization of BER in different modulation schemes: pulse position modulation, direct-sequence spread-spectrum, and frequency-hopping spread-spectrum.

The use of ray-tracing techniques to solve the rayobstacle equation system will further improve the calculation time in complex environments with furniture, curved surfaces, people, textures, etc. Also, the system can take advantage of the capabilities of new, cheap accelerating 3-D graphics cards, which include specialized hardware to solve ray-tracing and the illumination equations. Knowing the contribution to the received signal from any scatterer point has allowed us to improve the speed of the simulation dramatically.

\section{Acknowledgments}

This work has been supported by the Spanish Government (CICYT, TIC99-0932-C02-0, and 1FD97-1522) and TECNASA.

\section{References}

1. A. Santamaria and F.J. Lopez-Hernandez, Wireless LAN Systems, Chap. 1, Artech House, Boston (1994). 2. J.R. Barry, Wireless Infrared Communications, Chap. 4, Kluwer, Bos-
ton (1994).

3. F.J. Lopez-Hernandez and M.J. Betancor, "DUSTTN: a novel algorithm for the calculation of the impulse response on IR wireless indoor channels," Electron. Lett. 33(21), 1804-1805 (1997).

4. R. Perez-Jimenez, J. Berges, and M.J. Betancor, "Statistical model for the impulse response on infrared indoor diffuse channels," Electron. Lett. 33(15), 1298-1300 (1997). 5. O.N. Stavroudis, The Optics of Rays, Wavefronts, and Caustics, Chap.
6, Academic Press, New York (1972).

6. F.J. Lopez-Hernandez, R. Perez-Jimenez, and A. Santamaria, “Monte Carlo calculation of impulse response on diffuse IR wireless indoor channels," Electron. Lett. 34(12), 1260-1262 (1998).

. F.J. Lopez-Hernandez, R. Perez-Jimenez, and A. Santamaria, "Modified Monte Carlo scheme for high-efficiency simulation of the impulse response on diffuse IR wireless indoor channels," Electron. Let. 34(19), 1819-1820 (1998). 
López-Hernández, Pérez-Jiménez, and Santamaría: Ray-tracing algorithms ...

Francisco J. López-Hernandez received his MS and $\mathrm{PhD}$ from the ETSIT-UPM in 1978 and 1984, respectively. He is currently a professor at the UPM. His research interests are in the field of the wireless infrared communications, indoor channel simulation, and laser-based instrumentation devices.

Rafael Pérez-Jiménez received his MS in 1991 from the Universidad Politécnica de Madrid, and his PhD in 1995 from the Univer- sidad de Las Palmas de Gran Canaria (ULPGC), both in Spain. He is now a professor at the ULPGC. His current research interests are in IR indoor channel characterization and the design of IR systems for indoor communications.

Asunción Santamaría received her MS and PhD degrees from the ETSIT-UPM in 1989 and 1994, respectively. She is currently a professor at the UPM. Her research interests are in wireless infrared networking and wireless LAN protocol analysis. 